\title{
Dynamic Oligopoly with Sticky Prices: Closed-Loop, Feedback and Open-Loop Solutions
}

\author{
Roberto Cellinix - Luca Lambertini\# \\ §Dipartimento di Economia e M etodi Quantitativi \\ Università di Catania \\ Corso Italia 55, 95129 Catania, Italy \\ fax 39-095-370574 \\ e-mail cellini@mbox.unict.it \\ \#Dipartimento di Scienze Economiche \\ Università degli Studi di Bologna \\ Strada Maggiore 45 \\ 40125 Bologna, Italy \\ fax + 39-051-2092664 \\ e-mail lamberti@spbo.unibo.it
}




\begin{abstract}
A bstract
We investigate a dynamic oligopoly game with price adjustments. We show that the subgame perfect equilibria are characterised by larger output and lower price levels then the open-loop solution. The individual (and industry) output at the closed-loop equilibrium is larger than its counterpart at the feedback equilibrium. Therefore, ..rms prefer the open-loop equilibrium to the feedback equilibrium, and the latter to the closed-loop equilibrium. The opposite applies to consumers.
\end{abstract}

J EL Classi..cation: D43, D92, L 13

K eywords: dixerential games, loop decision rules, subgame perfection 


\section{Introduction}

The aim of this note consists in assessing comparatively the properties of open-loop, feedback and closed-loop memoryless equilibria in a dynamic oligopoly model with price dynamics ..rst introduced by Simaan and Takayama (1978) and then extended by Fershtman and Kamien (1987).

B roadly speaking, the main dixerence between the open-loop equilibrium on one side and the feedback and closed-loop equilibria on the other, is that the former does not take into account strategic interaction between players through the evolution of state variables over time and the associated adjustment in controls. Under the open-loop rule, players choose their respective plans at the initial date and commit to them forever. Therefore, in general, open-loop equilibria are not subgame perfect, in that they are only weakly time consistent because players make their action 'by the clock' only. ${ }^{1}$

A further distinction can be made between the closed-loop equilibrium and the feedback equilibrium, which are both strongly time consistent and therefore subgame perfect because, at any date $i$, players decide 'by the stock' of all state variables. However, while the closed-loop memoryless equilibrium takes into account the initial and current levels of all state variables, ${ }^{2}$

\footnotetext{
${ }^{1}$ For an exhaustive discussion of this issue, see ch. 6 in B aşar and Olsder (1982, 19952). However, there are classes of games where open-loop equilibria are subgame perfect. See Clemhout and Wan (1974); Reinganum (1982); M ehlmann and Willing (1983); Dockner, Feichtinger and J ørgensen (1985); Fershtman (1987). For an overview, see Mehlmann (1988); Fershtman, K amien and Muller (1992).

${ }^{2} \mathrm{~T}$ he information set associated with the closed-loop decision rule can take several forms. One consists in the level of the state variable(s) at the intial and current dates. This is usually de..ned as the closed-loop memoryless decision rule. A nother consists in the whole path of state variable(s) from the initial date to the present time. This is de.ned as closed-loop perfect state information rule.
} 
the feedback equilibrium accounts for the accumulated stock of each state variable at the current date. If one player decide according to the feedback rule, then it is optimal for the others to do so as well. Hence, the feedback equilibrium is a closed-loop equilibrium, while the opposite is not true in general. ${ }^{3}$

We extend the analysis of Fershtman and Kamien (1987) to investigate the open-loop, closed-loop memoryless and feedback equilibria of an industry with more than two players. Then, we characterise the closed-loop equilibrium for this market, to show the following results: (i) both subgame perfect equilibria involve a larger production and a lower price as compared to the open-loop solution; (ii) the steady state price and output levels are, respectively, higher and lower in the closed-loop equilibrium than in the feedback equilibrium. Property (i) can be reformulated by saying that, if ..rms are unable to initially commit to a given output plan for the whole time horizon, then subgame perfection entails overproduction (for analogous results see Spence, 1979; and Reynolds, 1987). Property (ii) suggests that the feedback rule allows ..rms to reduce overproduction as compared to the closed-loop rule, precisely because according to the feedback rule they look exclusively at the current level of the state variable.

The remainder of the paper is organised as follows. The model is laid out in section 2. Sections 3 and 4 , which illustrate the open-loop and the feedback equilibria, are simply the extension of Fershtman and Kamien's analysis to the oligopoly case. The closed-loop equilibrium is analysed in section 5. A comparative assessment of steady states is given in section 6 . Section 7 concludes the paper.

\footnotetext{
${ }^{3}$ For an exhaustive exposition of the dixerence among these equilibrium solutions, see B aşar and Olsder (1982, pp. 318-327, and chapter 6, in particular Proposition 6.1).
} 


\section{The setup}

Probably the simplest way to think about the dynamics of market interaction consists in assuming that prices evolve over time according to some acceptable rules. That is, it consists in taking price as the state variable. This is the problem analysed in Simaan and Takayama (1978) and Fershtman and Kamien (1987). ${ }^{4}$ Here, we present a generalisation of Fershtman and Kamien's setup to the case of $\mathrm{N}$..rms. ${ }^{5}$

Consider an oligopoly where, at any t $2[0 ; 1) ; \mathrm{N}$..rms produce quantities $q_{i}(t) ;$ i 2 f $1 ; 2 ; \cdots: N$ g; of the same homogeneous good at a total cost $C_{i}(t)=c q_{i}(t) i \frac{1}{2}\left[q_{i}(t)\right]^{2} ; c>0$ :

In each period, market demand determines the price level $p(t)=A_{i}$ ${ }_{i=1}^{P} q_{i}(t)$ : In general, however, $\boldsymbol{p}(t)$; will dixer from the current price level $p(t)$; since there is price stickness, and price moves according to the following equation:

$$
\frac{d p(t)}{d t} \cdot \dot{p}(t)=s f p(t) ; p(t) g
$$

Notice that the dynamics described by (1) establishes that price adjusts proportionately to the dixerence between the price level given by the inverse demand function and the current price level, the speed of adjustment being determined by the constant s; with $0<\mathrm{s}<1$.T his amounts to saying tha the price mechanism is sticky, that is, ..rms face menu costs in adjusting

\footnotetext{
${ }^{4}$ See also Mehlmann (1988, ch. 5) for an exhaustive exposition of both contributions, and Fershtman and Kamien (1990), and T sutsui and M ino (1990) for further results on the same model, in the case of a ..nite horizon.

${ }^{5} \mathrm{An}$ interesting application of this model to the analysis of adverting strategies is in Piga (2000). Trade policy issues are investigated by Dockner and Haugh (1990, 1991).
} 
their price to the demand conditions deriving from consumers' preferences: they may not (and, in general, the will not) choose outputs so that the price reaches immediately $\boldsymbol{p}(\mathrm{t})$ :

The instantaneous pro..t function of ..rm i is:

$$
1 / 4(t)=q_{i}(t) \oplus p(t) \text { i } \quad c_{i} \frac{1}{2} q_{i}(t)^{\circ}:
$$

Hence, the problem of ..rm i is:

$$
\max _{q(t)} J_{i}={ }_{0}^{Z_{1}} e^{i^{1 / 2}} q(t) \phi p(t) i \quad c i \frac{1}{2} q_{i}(t)^{\prime} d t
$$

subject to ( 1 ) and to the conditions $p(0)=p_{0}$; and $p(t), 0$ for all t $2[0 ; 1]$ :

We solve the problem by considering -in turn- the open-loop solution, the feedback solution and the closed-loop memoryless solution.

\section{The open-loop solution}

Here we look for the open-loop Nash equilibrium, i.e., we examine a situation where ..rms commit to a production plan at $\mathrm{t}=0$ and stick to that plan forever.

The Hamiltonian function is:

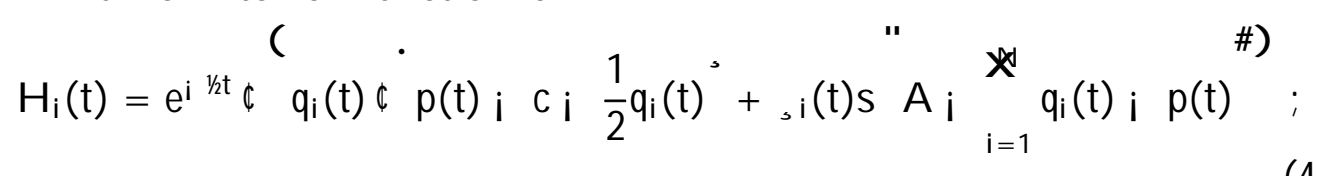

where ${ }_{i}(t)={ }^{1}{ }_{i}(t) e^{1 / 2}$; and ${ }^{1}{ }_{i}(t)$ is the co-state variable associated to $p(t)$ : The supplementary variable, $i(t)$ is introduced to ease calculations as well as the remainder of the exposition. In the remainder of the paper, superscript $\mathrm{OL}$ indicates the open-loop equilibrium level of a variable. The outcome of the open-loop game is summarised by: 
Proposition 1 At the open-loop Nash equilibrium, the steady state levels of the price and the individual output are:

$$
\begin{aligned}
& p^{O L}=A i N q^{O L} ; \\
& q^{O L}=\frac{(A ; C)(S+1 A}{(s+1 / A(1+N)+S}:
\end{aligned}
$$

The pair ${ }^{\mathrm{n}} \mathrm{p}^{\mathrm{OL}} ; \mathrm{q}^{\mathrm{OL}}{ }^{\mathrm{O}}$ is a saddle point.

Proof. Consider the ..rst order condition (FOC) w.r.t. $q_{i}(t)$; calculated using (4):

$$
\frac{@_{H_{i}}(t)}{@_{i}(t)}=p(t) ; \quad c ; \quad q_{i}(t) i, i(t) s=0 \text { : }
$$

This yields the optimal open-loop output for ..rm i; as follows: ${ }^{6}$

$$
q_{i}(t)=\begin{array}{ll}
8 \\
\gtrless
\end{array} p(t) i C i, i(t) s \quad \begin{array}{ll}
\text { if } p(t)>c+, i(t) s \\
\text { otherwise. }
\end{array}
$$

The remaining conditions for optimum are:

$$
\begin{aligned}
& \left.i \frac{@ H_{i}(t)}{@(t)}=i q_{i}(t)+, i(t) s=\frac{@_{i}(t)}{@ t}\right) \frac{@_{j}(t)}{@ i}=, i(t)\left(s+{ }^{1} / A i q_{i}(t) ;\right. \\
& \lim _{t ! 1}{ }^{1}{ }_{i}(t) \phi p(t)=0:
\end{aligned}
$$

Dixerentiating (6) and using (7), we obtain:

$$
\frac{d q_{i}(t)}{d t} \cdot \dot{q}(t)=\frac{d p(t)}{d t} ; s\left[(1 / 2+s), i(t) ; q_{i}(t)\right]:
$$

Now, substitute into (9) (i) $d p=d t=s f(t)$; $p(t) g$; with $p(t)=A$; $N q(t)$; where a symmetry assumption is introduced for individual ..rm's output; and (ii) $s_{s}(t)=p(t)$ i $c$ i $q(t)$ from (6). This yields:

$$
\frac{d q(t)}{d t}=s A+(s+1 / A c i \quad(2 s+1 / A p(t)+[s(1 ; N)+s+1 / 3 q(t)
$$

\footnotetext{
${ }^{6}$ In the remainder, we consider the positive solution. Obviously, the derivation of the steady state entails non-negativity constraints on price and quantity, that we assume to be satis..ed.
} 
Note that $d q(t)=d t=0$ is a linear relationship between $p(t)$ and $q(t): T$ his, together with $\mathrm{dp}(\mathrm{t})=\mathrm{dt}=0$; also a linear function, fully characterise the steady state of the system. The dynamic system can be immediately rewritten in matrix form as follows:

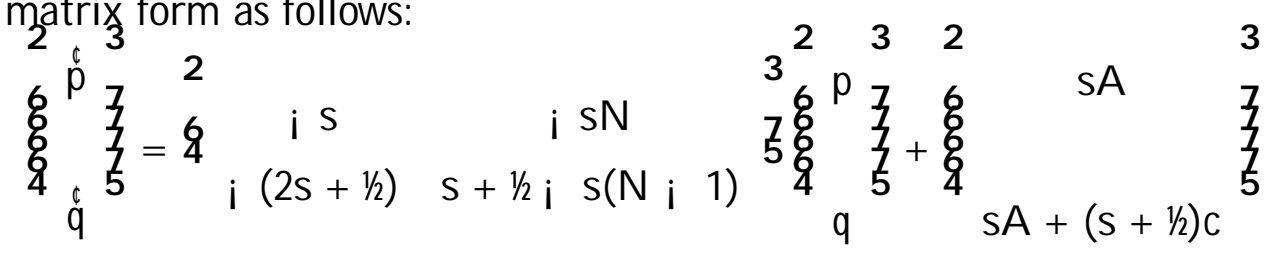

As the determinant of the above $2 £ 2$ matrix is negative, the equilibrium point is a saddle, with

$$
q^{O L}=\frac{(A ; C)(s+1 / A}{(s+1 / A(1+N)+s} ; p^{O L}=A ; N q^{O L} \text { : }
$$

This concludes the proof.

As in the duopoly case described by Fershtman and Kamien (1987 pp. 1159-61), also here the static Cournot-Nash equilibrium price and output ${ }^{\mathrm{C}} \mathrm{p}^{\mathrm{CN}} ; \mathrm{q}^{\mathrm{CN}}{ }^{\mathrm{O}}$ obtain from (12), in the limit, when $1 / 2$ ! 0 or $\mathrm{s} ! 1$ : For all positive levels of the discount rate and for any ..nite speed of adjustment, the static Cournot price (output) is higher (lower) than the open-loop equilibrium price (output).

\section{The feedback solution}

In this section, we extend the analysis of the feedback solution investigated by Fershtman and Kamien (1987) to the case of N ..rms. Using Bellman's value function approach, the feedback solution must satisfy the following set of Hamilton-B ellman-J acobi equations (see Starr and Ho, 1989):

$1 / D_{i}(p(t))=\max _{q(t)} q_{i}(t) \notin p(t) ; c_{i} \frac{1}{2} q_{i}(t)+\frac{\left(V_{i}(p(t))\right.}{@ p(t)} s A_{i}{ }_{i=1}^{N} q_{i}(t) i p(t)$ 
where $V_{i}(p(t))$ is the value function for ..rm $i$ : Hereinafter, the indication of time will be dropped to ease the exposition. Given the linear-quadratic form of the maximand, we follow Fershtman and Kamien (1987), and propose the quadratic value function:

$$
V_{i}(p)=\frac{k_{i} p^{2}}{2}+h_{i} p+g_{i}
$$

so that

$$
\frac{@ \mathrm{~V}_{i}(p)}{@ p}=k_{i} p+h_{i}:
$$

Henceforth, the superscript $F$ stands for feedback. The outcome of the game is summarised by:

Proposition 2 At the feedback Nash equilibrium, the steady state levels of the price and the individual output are:

$$
\begin{aligned}
& p^{F}=\frac{A+N c i, \bar{h} s}{N 1 i k s+1} ;
\end{aligned}
$$

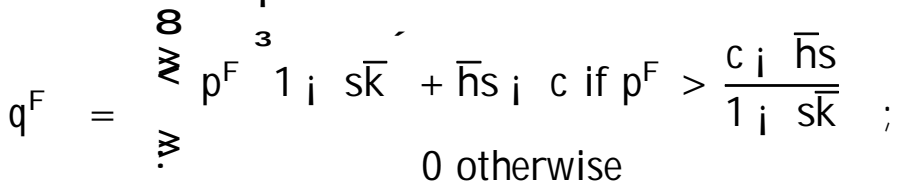

where

$$
\begin{aligned}
& \bar{h}=\frac{{ }_{3} c i s(a i N c) \bar{k}}{1 / 2+s \overline{k s} i 2 N \overline{k s}+N+1} ; \\
& \bar{k}=\frac{1 / 2+2 s(N+1) i \frac{q}{1 / 2+4 s\left(1 / 2+N 1 / 2+2 s+s N^{2}\right)}}{2(2 N i 1) s^{2}}:
\end{aligned}
$$

Proof. Taking the FOC w.r.t.q; ; we obtain:

$$
q_{i}^{F}=p_{i} c i s \frac{@ V_{i}(p)}{@ p}=p_{i} c i s\left(k_{i} p+h_{i}\right) ;
$$


where we invoke the symmetry conditions $g_{i}=g ; k_{i}=k$ and $h_{i}=h$; so that $q_{i}=q$ for all $i:$ On the basis of (16) and (1), we ..nd:

$$
p^{F}=\frac{A+N(c ; h s)}{N(1 ; k s)+1} ;
$$

where $\mathrm{h}$ and $\mathrm{k}$ can be calculated by the following procedure. We can rewrite (13) as:

$$
1 / 4 /(p) ; \max 1 / 4+\frac{@(p)}{@ p} s \frac{d p^{\prime}}{d t}=0 \text {; }
$$

that is:

$$
{ }_{1} p^{2}+{ }_{2} p+{ }_{3}=0 \text {; }
$$

where

$$
\begin{gathered}
-_{1}=\frac{k[1 / 2+s(2+2 N+k s i 2 k s N)] i 1}{2} ; \\
-c_{2}=h^{2}(1 / 2+s+s N) \text { i ks }(A+N c+h s i 2 h s N) ; \\
-{ }_{3}=\frac{2 g^{1} / 2 i c^{2}+h s(2 A+2 N c+h s i 2 h s N)}{2}:
\end{gathered}
$$

Expression (19) is satis..ed if expressions (20), (21) and (22), i.e., coec cients ${ }^{-}{ }_{1} ;{ }_{2}$ and ${ }^{-}{ }_{3}$ are simultaneously zero. This makes up a system of three equations in three unknowns, $\mathrm{fg} ; \mathrm{h} ; \mathrm{kg}$; with the following solutions:

$$
\begin{aligned}
& g=\frac{c^{2} i h s(2 A+2 N c+h s i 2 h s N)}{2^{1 / 2}} ; \\
& h=\frac{{ }_{3}^{c} \text { i s }\left(a_{i} N c\right) k}{1 / 2+s k s \text { i } 2 N k s+N+1}, \bar{h} \text {; }
\end{aligned}
$$

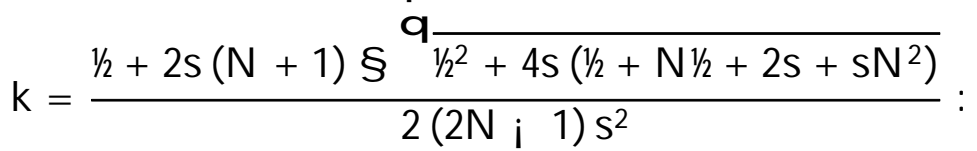

We choose the smaller solution for $\mathrm{k}$ in (25), which yields - when $\mathrm{N}=2$ - the same expression as in Fershtman and Kamien (1987, Theorem 2, p. 1157). This establishes that

$$
\bar{k}=\frac{1 / 2+2 s(N+1) i^{q} \frac{1 / 2+4 s\left(1 / 2+N^{1 / 2}+2 s+s N^{2}\right)}{1 / 2 N i 1) s^{2}}}{2(2 N)}
$$


This concludes the proof.

\section{The closed-loop solution}

The closed-loop memoryless solution remains to investigate. We use superscript $C L$ to denote the closed-loop equilibrium levels of the relevant variables. The Hamiltonian of ..rm i is given by (4), and the outcome is summarised by the following:

Proposition 3 At the closed-loop Nash equilibrium, the steady state levels of the price and the individual output are:

$$
\begin{aligned}
& p^{C L}=A i N q^{C L} ; \\
& q^{C L}=\frac{(A ; C)(1 / 2+s N)}{(N+1)^{1 / 2+\left(N^{2}+N+1\right) S}:}
\end{aligned}
$$

The pair ${ }^{n} p^{C L} ; q^{C L}{ }^{\circ}$ is a saddle point.

Proof. The ..rst order condition w.r.t. q; calculated using (4), obviously coincide with condition (5) calculated in the open-loop case:

$$
\frac{\mathfrak{a H}_{i}}{\mathfrak{Q}_{\mathrm{q}}}=p_{\mathrm{i}} \mathrm{c}_{\mathrm{i}} q_{\mathrm{i}} \mathrm{i}, \mathrm{i} \mathrm{s}=0 \text { : }
$$

This yields the closed-loop output for ..rm i; as follows (again, in the remainder we shall consider only the positive solution):

$$
q_{i}^{C L}=\begin{array}{lc}
\stackrel{8}{\gtrless} p_{i} C i, i s & \text { if } p>C+, i s \\
>0 & \text { otherwise. }
\end{array}
$$

The remaining conditions for optimum are:

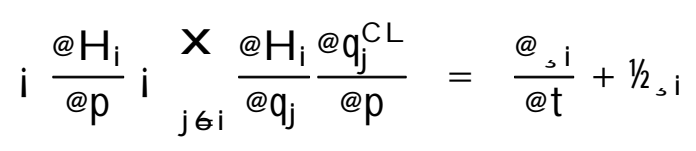


Now consider that

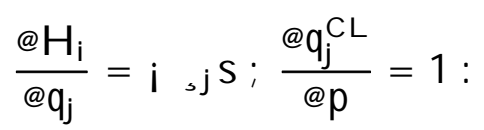

Therefore:

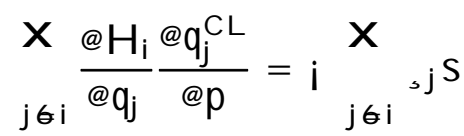

is the additional term in the co-state equation, characterising the strategic interaction among ..rms, which is not considered by de..nition in the openloop solution (see, e.g., Driskill and McCaxerty, 1989). Equation (29) may

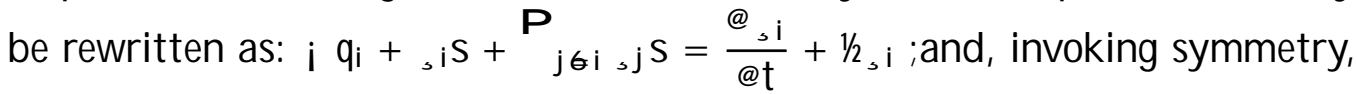
one obtains:

$$
\frac{@}{\mathrm{~d} t}=\mathrm{iq}+,(1 / 2+\mathrm{N} \mathrm{s}) \text { : }
$$

Then we have the transversality condition:

$$
\lim _{t ! 1}{ }^{1}{ }_{i} \phi p=0:
$$

Dixerentiating (28) w.r.t. time and using (32), we obtain:

$$
\frac{d q}{d t}=\frac{d p}{d t} \text { i } s\left[(1 / 2+w) \text {, i i } q_{i}\right] \text { : }
$$

Now, substitute into (34) the expressions (i) $d p=d t=s f p_{i} p g$; with $p(t)=$ A i $\mathrm{N}$ q; where a symmetry assumption is introduced for individual ..rm's output; and (ii) s, = p i ci q which obtains from (27). This yields:

$$
\frac{d q}{d t}=1 / 2(c ; p+q)+s[A ; p+q ; N(p ; c)]
$$

As in the open-loop case, $d q=d t=0$ is a linear relationship between $p$ and $q$ : This, together with $\mathrm{dp}=\mathrm{dt}=0$; which is also a linear function, yields

$$
\begin{aligned}
& p^{C L}=A ; \frac{N(A ; C)(1 / 2+s N)}{(N+1)^{1 / 2}+\left(N^{2}+N+1\right) s} ; \\
& q^{C L}=\frac{(A ; C)(1 / 2+s N)}{(N+1)^{1 / 2+\left(N^{2}+N+1\right) s}:}
\end{aligned}
$$


as the unique steady state of the system. ${ }^{7}$ The dynamic system can be immediately rewritten in matrix form to verify that the pair ${ }^{\mathrm{n}} \mathrm{p}^{\mathrm{CL}} ; \mathrm{q}^{\mathrm{CL}}{ }^{\mathrm{O}}$ is a saddle point. The proof of this is omitted for the sake of brevity.

\section{Comparative assessment of steady states}

Now we can compare the steady state levels of price and individual output in the three cases analysed above, as well as in the static case. The following result emerges.

Proposition 4 For all s $2[0 ; 1]$ and all N $2[1 ; 1)$;

$$
\begin{aligned}
& q^{C L}>q^{F}>q^{O L}>q^{C N} \\
& p^{C N}>p^{O L}>p^{F}>p^{C L}:
\end{aligned}
$$

The proof is straightforward. In words, con..ning our attention to the equilibria of the dynamic setting, all subgame perfect equilibria entails a higher (individual and industry) output and a lower market price than the open-loop equilibrium (which is not subgame perfect). Therefore, Proposition 4 produces a relevant Corollary:

Corollary 1 From the ..rms' viewpoint, the open-loop equilibrium is preferred to both the feedback equilibrium and the closed-loop memoryless equilibrium. On the contrary, the closed-loop memoryless equilibrium is socially preferred to the feedback and open-loop equilibria.

Fershtman and Kamien (1987, pp. 1159-61) also investigate the properties of the limit games, where the speed of adjustment s tends to in..nity or

\footnotetext{
${ }^{7}$ Of course by "unique" we mean the only steady state with positive price and outputs.
} 
$1 / 2$ becomes nil. They establish that, in such cases, the open-loop equilibrium coincides with the $\mathrm{Nash}$ equilibrium of the static game. However, considering an in..nitely high speed of price adjustment seems more a mathematical curiosum than a theoretically relevant case, in that whenever $s>1$; the instantaneous change in price is larger than the error $p(t)$; $p(t)$ : If we con..ne to 2 [0; 1]; we obtain the following Corollary to Proposition 1 :

Corollary 2 When $s=1$;

$$
q^{O L}=\frac{(A ; C)(1+1 / A}{(N+1)(1+1 / A+1}
$$

which is larger than the static Cournot- $N$ ash output

$$
q^{C N}=\frac{A_{i} C}{N+2}
$$

for all $N, 1$ and all $1 / 2>0$ :

In the limit, as $1 / 2$ ! $0 ; q^{O L} ! q^{C N}$ for all admissible $N$ and $\mathrm{s}$ :

F inally, one can check what happens to the steady state levels of output as $\mathrm{N}$ tends to in..nity, to verify the following Corollary to Proposition 4 :

Corollary 3 As the number of ..rms becomes in...nitely large, optimal individual output tends to zero indipendently of the solution concept.

Therefore, as the market becomes perfectly competitive, open-loop, closedloop and feedback solutions coincide with the static Cournot-Nash solution, which is itself reproducing the perfectly competitive outcome.

\section{Concluding remarks}

We have investigated the properties of a dynamic oligopoly game with sticky prices. The foregoing analysis shows that subgame perfection always entails larger output and lower price levels in steady state, as compared to 
the weakly time consistent open-loop solution. In particular, the individual and industry output associated to the closed-loop equilibrium is larger than its counterpart at the feedback equilibrium. T wo further (and related) remarks are in order. First, the foregoing analysis highlights that the larger the relevant information set, the larger the overproduction compared to the (commitment) open-loop equilibrium. The second is that among the subgame perfect solution concepts, the feedback rule appears to be able to minimise overproduction. Accordingly, while ..rms would prefer the open-loop equilibrium to the feedback equilibrium, and the latter to the closed-loop equilibrium, the opposite holds from the social standpoint. 


\section{R eferences}

[1] Başar, T. and G.J. Olsder (1982, 1995²), Dynamic Noncooperative Game Theory, San Diego, A cademic Press.

[2] Clemhout, S. and H.Y. Wan, J r. (1974), "A Class of Trilinear Dixerential Games", J ournal of Optimization Theory and A pplications, 14, 419-24.

[3] Dockner, E.J . and A.A. Haug (1990), "Tarias and Quotas under Dynamic Duopolistic Competition", J ournal of International Economics, 29, 147-59.

[4] Dockner, E.J . and A.A. Haug (1991), "The Closed Loop Motive for Voluntary Export Restraints", Canadian J ournal of Economics, 3, 67985.

[5] Dockner, E.J., G. Feichtinger and S. J ørgensen (1985), "Tractable Classes of Nonzero-Sum Open-Loop Nash Dixerential Games: Theory and Examples", J ournal of Optimization Theory and Applications, 45, 179-97.

[6] Driskill, R. and S. M cCaxerty (1989), “Dynamic Duopoly with Adjustment Costs: A Diperential Game Approach", J ournal of E conomic Theory, $69,324-38$.

[7] Fershtman, C. (1987), "Identi..cation of Classes of Dixerential Games for W hich the Open-Loop is a degenertaed Feedback Nash Equilibrium", J ournal of Optimization Theory and Applications, 55, 217-31.

[8] Fershtman, C. and M.I. Kamien (1987), “Dynamic Duopolistic Competition with Sticky Prices", Econometrica, 55, 1151-64. 
[9] Fershtman, C. and M.I. Kamien (1990), "Turnpike Properties in a Finite-Horizon Dixerential Game: Dynamic Duopoly with Sticky Prices", International Economic Review, 31, 49-60.

[10] Fershtman, C., M. Kamien and E. Muller (1992), "Integral Games: Theory and A pplications", in Feichtinger, G. (ed.), Dynamic Economic M odels and Optimal Control, A msterdam, North-Holland, 297-311.

[11] Mehlmann, A. (1988), Applied Dixerential Games, New York, Plenum Press.

[12] Mehlmann, A . and R. W illing (1983), “On Nonunique Closed-Loop Nash Equilibria for a Class of Dixerential Games with a Unique and Degenerate Feedback Solution", J ournal of Optimization Theory and Applications, 41, 463-72.

[13] Piga, C. (2000), "Competition in a Duopoly with Sticky Price and Advertising", International J ournal of Industrial Organization, 18, 595-614.

[14] Reinganum, J. (1982), "A Class of Dixerential Games for Which the Closed Loop and Open Loop Nash Equilibria Coincide", J ournal of Optimization Theory and Applications, 36, 253-62.

[15] Reynolds, S.S. (1987), "Capacity Investment, Preemption and Commitment in an In..nite Horizon Model", International Economic Review, 28, 69-88.

[16] Simaan, M. and T. Takayama (1978), "Game Theory Applied to Dynamic Duopoly Problems with Production Constraints", Automatica, 14, 161-6. 
[17] Spence, A. M. (1979), "Investment Strategy and Growth in a New Market", Bell J ournal of Economics, 10, 1-19.

[18] Starr, A.W. and Y.C. Ho (1969), "Nonzero-Sum Dixerential Games", J ournal of Optimization Theory and Applications, 3, 184-208.

[19] Tsutsui, S. and K. Mino (1990), "Nonlinear Strategies in Dynamic Duopolistic Competition with Sticky Prices", J ournal of E conomic Theory, 52, 136-61. 